\title{
Comparison of Laparoscopic and Open Surgery for Colorectal Cancer in Patients with Severe Comorbidities
}

\author{
MASAKATSU NUMATA ${ }^{1}$, SHO SAWAZAKI ${ }^{1}$, JUNYA MORITA $^{1}$, YUKIO MAEZAWA ${ }^{1}$, SHINYA AMANO ${ }^{1}$, \\ TORU AOYAMA ${ }^{1}$, TSUTOMU SATO ${ }^{1}$, TAKASHI OSHIMA ${ }^{1}$, HIROYUKI MUSHIAKE ${ }^{1}$, \\ NORIO YUKAWA ${ }^{1}$, MANABU SHIOZAWA ${ }^{2}$, YASUSHI RINO ${ }^{1}$ and MUNETAKA MASUDA ${ }^{1}$ \\ ${ }^{1}$ Department of Surgery, Yokohama City University, Yokohama, Japan; \\ ${ }^{2}$ Department Gastroenterological Surgery, Kanagawa Cancer Center Hospital, Yokohama, Japan
}

\begin{abstract}
Background/Aim: To evaluate the safety of laparoscopic colorectal cancer surgery for patients with severe comorbidities. Patients and Methods: A total of 203 consecutive patients with severe comorbidities who underwent resection for colorectal cancer were retrospectively divided into laparoscopic and open primary resection groups. An age-adjusted Charlson comorbidity index $\geq 6$ was considered as severe comorbidity. Results: Blood loss (31 g vs. $207 \mathrm{~g}, p<0.01)$ and total postoperative complications $(10.0 \%$ vs. $27.5 \%, p<0.01)$ in the laparoscopic group were significantly decreased compared to the open group. Incidence of postoperative ileus $(0.0 \% \mathrm{vs} .7 .2 \%$, $p=0.06$ ) and length of postoperative hospital stay (11 days vs. 14 days, $p=0.08$ ) in the laparoscopic group were improved, though not significantly, compared to the open group. Conclusion: Laparoscopic resection for patients with severe comorbidities is safe, and is associated with a lower rate of overall operative complications compared to open surgery.
\end{abstract}

The benefits of laparoscopic surgery for colorectal cancer have been well established. These benefits include earlier recovery of bowel function, a lower rate of intra-abdominal adhesions, reduced analgesic requirements, decreased hospital stay, and a reduced morbidity rate (1-5). However, laparoscopic surgery performed with pneumoperitoneum and using the Trendelenburg position is associated with adverse effects on hemodynamic and respiratory status, such as increasing systemic vascular resistance, decreasing the ejection fraction, and respiratory compliance (6). Due to

Correspondence to: Masakatsu Numata, Department of Surgery, Yokohama City University, 3-9 Fukuura, Kanazawa-ku, Yokohama, Kanagawa 236-0004, Japan. Tel: +81 457872800, Fax: +81 457860226, e-mail: masakatsunumata@hotmail.co.jp

Key Words: Colorectal cancer, laparoscopic surgery, comorbidity, age-adjusted Charlson comorbidity index. concern for these adverse aspects, laparoscopic surgery is not generally considered for patients with a severe comorbidity. Thus, there have been few reports regarding the outcomes of patients with severe comorbidities undergoing laparoscopic colorectal surgery, and only a limited number of studies have tried to address the problem.

The Charlson Comorbidity Index (CCI) is comprised of 19 disease conditions, each assigned with a weighted score (7), and has been validated as an effective predictor of patient outcome in colorectal cancer (8-11). The age-adjusted CCI (ACCI) is a modification that considers age as an additional comorbidity factor (12) (Table I). ACCI has also been widely used as a predictive tool in various diseases (1316). According to a previous study, an ACCI score $\geq 6$ was considered a severe comorbidity $(13,17)$.

In this study, the safety of laparoscopic colorectal resection in patients with severe comorbidities was examined, comparing their outcomes with a similar cohort who underwent open resection in the same period. ACCI was used to categorize the patients based on severity of the comorbidities.

\section{Patients and Methods}

Patient enrollment. This study included consecutive patients who had severe comorbidities who underwent primary surgical resection for colorectal cancer in a university teaching hospital from April 2004 to March 2016. A total of 356 patients underwent primary resection for colorectal cancer in that period. A total of 39 patients were excluded because of multiple organ resection due to synchronous multiple cancers or metastasis (i.e.; synchronous hepatectomy or gastrectomy). Among the 317 remaining patients, $203(64.0 \%)$ had ACCI $\geq 6$, which was considered severe comorbidity. These 203 patients were then divided into those who had laparoscopic versus open primary resections (Figure 1).

The indication for laparoscopic surgery rather than open was not standardized, but generally was based on surgical experience, patient preference, and the size of the tumor. Patient data, which includes information regarding the preoperative assessment, operative outcomes, and postoperative complications, were retrospectively reviewed in a prospective database. 
Table I. Age-adjusted Charlson Comorbidity Index.

\begin{tabular}{ll}
\hline Score & Disease condition \\
\hline 1 & Myocardial infarction \\
& Congestive heart failure \\
& Peripheral vascular disease \\
& Cerebrovascular disease \\
& Dementia \\
& Chronic pulmonary disease \\
& Connective tissue disease \\
& Peptic ulcer disease \\
& Mild liver disease \\
& Diabetes without end-organ damage \\
& Hemiplegia \\
& Moderate or severe renal disease \\
& Diabetes with end-organ damage \\
& Tumor without metastasis \\
& Leukemia \\
& Lymphoma \\
& Moderate or severe liver disease \\
& Metastatic solid tumor \\
& Acquired immunodeficiency syndrome \\
& \\
6 & For each decade >40 years of age, \\
& a score of 1 is added. Maximum score is 4.
\end{tabular}

All study protocols were approved by our institutional review board (No. B170700003). The details of the study protocol were provided to patients through a notice board in the hospital and published on the hospital website.

Perioperative management. The perioperative management of patients was similar in both groups. The patients underwent bowel preparation with polyethylene glycol electrolyte solution and began a diet on the third day after surgery. All patients received intravenous cefmetazole from just before the start of the operation until the day after. Deep venous thrombosis prophylaxis included a perioperative antiembolic stocking and intraoperative pneumatic calf compression for patients without peripheral vascular disease. Postoperative complications were evaluated according to the Clavien-Dindo classification (18). The criteria for patient discharge in both groups included tolerance of the diet without nausea or vomiting, passage of flatus and stool, and sufficient recovery from any postoperative complications.

Operative techniques. All operations were performed, or directly supervised, by one of 6 experienced colorectal surgeons. For the laparoscopic procedure, first access to the abdomen was usually achieved via the umbilical port. Once pneumoperitoneum was established, 4 additional ports were placed. Vessel ligation with lymph node dissection was performed, followed by mobilization of the colon. The specimen was extracted via a 4- to $6 \mathrm{~cm}$ umbilical incision. Bowel anastomosis was performed intracorporeally with the doubling staple technique for colorectal anastomosis, and extracorporeally at the extraction site for ileocolic and colo-colonic anastomosis. For the open colorectal resection, a midline laparotomy was the access point of choice. Mobilization of the colon was followed by ligation of the

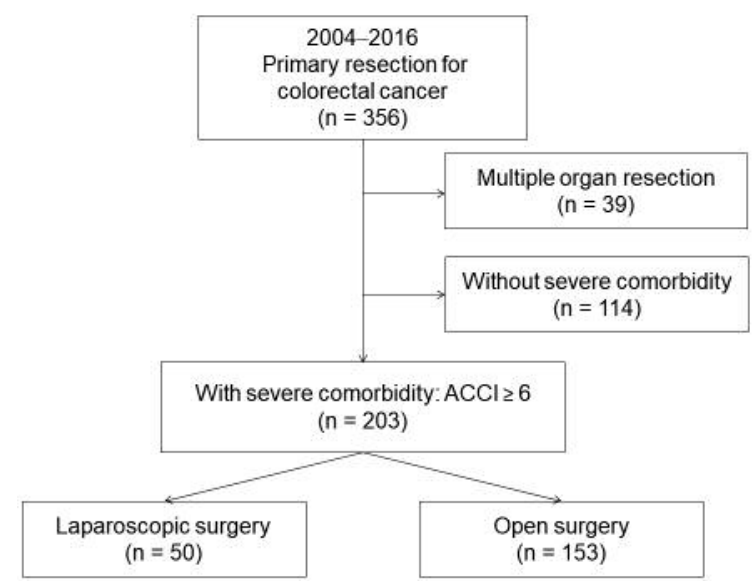

Figure 1. Consort diagram. ACCI: Age-adjusted Charlson Comorbidity Index.

vessels, bowel resection, and bowel anastomosis. Any incision $>8 \mathrm{~cm}$ was considered a conversion to open surgery.

Statistical analysis. The differences in patient characteristics, operative findings, and postoperative results were analyzed between the two groups. Comparisons between the laparoscopic and open group were made on an intent-to-treat basis. Chi-square and Fisher's exact tests were used for categorical variables. Student's $t$-test was used to compare continuous variables. All statistical analyses were performed with EZR (19) (Jichi Medical University, Saitama, Japan). All $p$-values were 2 -sided, and $p$-values less than 0.05 were considered statistically significant.

\section{Results}

The demographic characteristics of the two groups are presented in Table II. There were no meaningful differences in terms of age, sex, body mass index, American Society of Anesthesiologists (ASA) physical status classification, comorbidity details, serum albumin, serum carcinoembryonic antigen levels, location of the tumor, and TMN stage. The mean ACCI score was significantly lower (6 vs. 7, $p<0.01)$, and tumor diameter was significantly smaller (32 $\mathrm{mm} v \mathrm{~s}$. $45 \mathrm{~mm}, p<0.01)$ in the laparoscopic group compared with the open group.

The operative outcomes of the two groups are summarized in Table III. The rate of conversion to open surgery for the laparoscopic group was $8.0 \%$ (4 cases). The reasons for conversions were tumor invasion to an adjacent structure in 3 cases, and severe intra-abdominal adhesion in 1 case. There was no conversion as a result of physiological intolerance of pneumoperitoneum or the Trendelenburg position. Although operative time was longer $(247 \mathrm{~min} v s .181 \mathrm{~min}, p<0.01)$ in the laparoscopic group, blood loss (31 g vs. $207 \mathrm{~g}, p<0.01$ ) and postoperative complications $(10.0 \%$ vs. $27.5 \%, p=0.01)$ 
Table II. Demographic characteristics $(n=203)$.

\begin{tabular}{|c|c|c|c|}
\hline Parameters & Laparoscopic group $(n=50)$ & Open group $(n=153)$ & $p$-Value ${ }^{\mathrm{a}}$ \\
\hline Age (years), median (range) & $75(59-86)$ & $73(30-89)$ & 0.26 \\
\hline Gender & & & 0.82 \\
\hline Male & 19 & 92 & \\
\hline Female & 31 & 61 & \\
\hline BMI $\left(\mathrm{kg} / \mathrm{m}^{2}\right)$, median (range) & $21.6(15.4-37.7)$ & $21.9(14.8-34.2)$ & 0.80 \\
\hline ASA classification & & & 0.23 \\
\hline Class 2 & 14 & 27 & \\
\hline Class 3 & 36 & 124 & \\
\hline Class 4 & 0 & 2 & \\
\hline ACCI, median (range) & $6(6-13)$ & $7(6-16)$ & $<0.01$ \\
\hline \multicolumn{4}{|l|}{ Comorbidity $\mathrm{b}$} \\
\hline Cardiovascular disease & 28 & 82 & 0.87 \\
\hline Pulmonary disease & 13 & 45 & 0.72 \\
\hline Renal dysfunction & 1 & 12 & 0.19 \\
\hline Hepatic disease & 0 & 6 & 0.39 \\
\hline Diabetes mellitus & 12 & 26 & 0.29 \\
\hline Neurological disease & 7 & 29 & 0.52 \\
\hline Serum albumin (g/dl), median (range) & $4.2(3.1-4.8)$ & $4.0(1.5-5.0)$ & 0.66 \\
\hline CEA (ng/ml), median (range) & $3.3(0.3-56.8)$ & $5.6(0.2-7942)$ & 0.35 \\
\hline Location (colon/rectum) & $38 / 12$ & $100 / 53$ & 0.72 \\
\hline Tumor diameter $(\mathrm{mm})$, median (range) & $32(11-117)$ & $45(2-180)$ & $\leq 0.01$ \\
\hline TMN Stage 0/I/II/III/IV & $1 / 21 / 12 / 12 / 4$ & $7 / 19 / 46 / 37 / 44$ & $\overline{0.25}$ \\
\hline
\end{tabular}

BMI: Body mass index; ASA: American Society of Anesthesiologists physical status score; AACI: Age-adjusted Charlson Comorbidity Index, CEA:

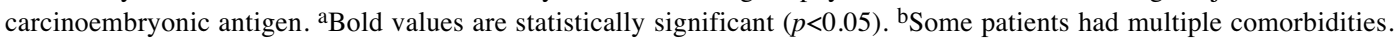

were significantly better compared to the open group. Compared to the open group, the rate of ileus $(0.0 \% \mathrm{vs.} 7.2 \%$, $p=0.06)$ and length of the postoperative hospital stay (11 days $v s .14$ days, $p=0.08$ ) tended to be better in the laparoscopic group, although not significant. Mortality was low in both groups $(0.0 \%$ vs. $0.7 \%, p=1.00)$.

\section{Discussion}

Laparoscopic surgery offers the benefit of faster recovery for patients. However, pneumoperitoneum with carbon dioxide is associated with potential adverse pathophysiological changes, including hypercapnia, reduced venous return, increased peak airway pressure, and decrease pulmonary compliance (6). It has been reported that raised intracranial pressure, severe myopia, and/or retinal detachment are absolute contraindications for laparoscopy, whereas bullous emphysema, history of spontaneous pneumothorax, and pregnancy are relative contraindications (20). Because of the concern for potential hazards, the recommendation criteria for laparoscopy in patients with severe comorbidities have not been well defined.

Tashiro et al. (21) stratified patient comorbidity by ASA score (ASA1, ASA2-3, or ASA4) and compared the short-term results after laparoscopic resection for colorectal cancer. They reported that the incidence of postoperative complications was not significantly different for the groups. Ichikawa et al. (22). also used the ASA score for assessing the severity of comorbidities in patients who underwent resection for colorectal cancer, then compared the complication rates in 3 groups: a laparoscopic group with ASA score of 3 (LAP3), laparoscopic group with ASA score <2 (LAP2), and the open group with ASA score of 3 (OP3). They found that the total complication rate in LAP3 was similar with that seen in LAP2, and significantly lower than that seen in OP3, and concluded that laparoscopic colorectal resection can be performed safely in patients with severe comorbidities. Stocchi et al. performed a case-control analysis of the safety of laparoscopic and open surgery procedures for colorectal cancer in patients aged over 75 years (23). They reported that laparoscopic surgery was associated with significantly fewer post-operative morbidities ( $14.3 \%$ for laparoscopic surgery $v s .33 .3 \%$ for open surgery; $p=0.04)$, and decreased length of hospital stay (6.5 vs. 10.2 days; $p<0.001)$, resulting in the determination that laparoscopic surgery is safe and beneficial for elderly patients compared with open colectomy.

As age was recognized as an independent factor for postoperative complications after colon cancer surgery (24), we used the ACCI to quantify base-line comorbidities. The ACCI is not just a weighted measure that reflects the severity of the comorbidity, but it also considers age as an 
Table III. Operative outcomes ( $n=203$ ).

\begin{tabular}{|c|c|c|c|}
\hline Parameters & Laparoscopic group $(n=50)$ & Open group $(n=153)$ & $p$-Value \\
\hline \multicolumn{4}{|l|}{ Operative outcomes } \\
\hline Operative time (min), median (range) & $247(126-459)$ & $181(57-585)$ & $<0.01$ \\
\hline Blood loss (g), median (range) & $31(0-1410)$ & $207(0-4850)$ & $<0.01$ \\
\hline Conversion to open surgery & $4(8.0 \%)$ & - & - \\
\hline Invasion to adjacent structure & $3(6.0 \%)$ & & \\
\hline Severe intra-abdominal adhesion & $1(2.0 \%)$ & & \\
\hline Postoperative complications ( $\geq \mathrm{CD}$ Grade II) & $5(10.0 \%)$ & $42(27.5 \%)$ & $\mathbf{0 . 0 1}$ \\
\hline \multicolumn{4}{|l|}{ Details of complications ${ }^{b}$} \\
\hline Wound infection & $0(0.0 \%)$ & $7(4.6 \%)$ & 0.19 \\
\hline Anastomotic leakage & $2(4.0 \%)$ & $6(3.9 \%)$ & 1.00 \\
\hline Ileus & $0(0.0 \%)$ & $11(7.2 \%)$ & 0.06 \\
\hline Abdominal abscess & $2(4.0 \%)$ & $7(4.6 \%)$ & 0.65 \\
\hline Cardiovascular complication & $0(0.0 \%)$ & $2(1.3 \%)$ & 1.00 \\
\hline Respiratory complication & $0(0.0 \%)$ & $1(0.7 \%)$ & 1.00 \\
\hline Delirium & $0(0.0 \%)$ & $1(0.7 \%)$ & 1.00 \\
\hline Urinary tract infection & $0(0.0 \%)$ & $1(0.7 \%)$ & 1.00 \\
\hline Cerebral stroke & $0(0.0 \%)$ & $1(0.7 \%)$ & 1.00 \\
\hline Sepsis & $0(0.0 \%)$ & $3(2.0 \%)$ & 1.00 \\
\hline Other complication & $1(2.0 \%)$ & $5(3.3 \%)$ & 1.00 \\
\hline Postoperative hospital stay (days), median (range) & $11(7-74)$ & $14(4-96)$ & 0.08 \\
\hline Mortality & $0(0.0 \%)$ & $1(0.7 \%)$ & 1.00 \\
\hline
\end{tabular}

CD: Clavien Dindo Classification. ${ }^{a}$ Bold values are statistically significant $(p<0.05)$. bSome patients had more than one complication.

important risk factor that can be used to evaluate the patient's general physical condition. Indeed, among all patients in this study, $41(20.1 \%)$ were classified as having an ASA score of 2 , and would have been excluded from analysis if the enrollment criterium was ASA class 3 or higher. As far as we know, there have been no studies that have used ACCI to categorize preoperative comorbidities, when comparing the short-term result after laparoscopic and open surgery for colorectal cancer.

In this study, the laparoscopic group had fewer overall postoperative complications. Specifically, the laparoscopic group had fewer wound infections and a lower rate of postoperative ileus, although these differences were not statistically significant. Previous reports have shown that laparoscopic surgery is superior to open surgery in terms of wound complication (25) and ileus (26) after colorectal surgery. Our study confirmed that this superiority was preserved even in patients with severe comorbidities. It has also been previously reported that laparoscopic surgery reduces surgical trauma, resulting in improved serum interleukin and cytokine responses compared with open surgery (27), that may contribute to the favorable outcome in the laparoscopic group observed in this study.

Importantly, our results show that laparoscopic surgery was well tolerated by patients with cardiovascular or pulmonary disease, and no patient required open conversion due of intolerance of pneumoperitoneum. In a review article regarding anesthesia for laparoscopy, it was reported that the adverse cardiopulmonary effect of pneumoperitoneum becomes clinically evident when intraabdominal pressure is more than $15 \mathrm{~mm} \mathrm{Hg}$. At a pressure of less than $12 \mathrm{~mm} \mathrm{Hg}$, the venous return is augmented by increased outflow of blood from the splanchnic venous bed without potential burden on the cardiopulmonary system (20). With careful monitoring and improvement in anesthetic techniques, laparoscopic surgery may be performed safely in cases with severe comorbidities.

This study has several limitations. The first limitation is that it is retrospective and nonrandomized in design. When comparing patient backgrounds, the median ACCI scores and tumor diameters were lower in the laparoscopic group than the open group. These differences could affect the favorable result observed for the laparoscopic group. Thus, the results of this study are not generally applicable to all patients with a severe comorbidity, but only for selected patients. The second limitation is the sample size. The present study collected the data from a university teaching hospital over a 12 -year period and identified 203 patients. Combined data from multiple centers are needed to confirm the results of the present study.

In conclusion, laparoscopic resection for patients with severe comorbidities is safe, and associated with a lower rate of complications compared to open surgery. A randomized retrospective study or case-matched large analysis is needed to confirm our results. 


\section{References}

1 Lacy AM, Garcia-Valdecasas JC, Delgado S, Castells A, Taura $\mathrm{P}$, Pique JM and Visa J: Laparoscopy-assisted colectomy versus open colectomy for treatment of non-metastatic colon cancer: A randomised trial. Lancet 359(9325): 2224-2229, 2002.

2 Clinical Outcomes of Surgical Therapy Study Group, Nelson H, Sargent DJ, Wieand HS, Fleshman J, Anvari M, Stryker SJ, Beart RW Jr, Hellinger M, Flanagan R Jr., Peters W and Ota D: A comparison of laparoscopically assisted and open colectomy for colon cancer. N Engl J Med 350(20): 2050-2059, 2004.

3 Veldkamp R, Kuhry E, Hop WC, Jeekel J, Kazemier G, Bonjer HJ, Haglind E, Pahlman L, Cuesta MA, Msika S, Morino M and Lacy AM: Laparoscopic surgery versus open surgery for colon cancer: Short-term outcomes of a randomised trial. Lancet Oncol 6(7): 477-484, 2005.

4 Guillou PJ, Quirke P, Thorpe H, Walker J, Jayne DG, Smith AM, Heath RM and Brown JM: Short-term endpoints of conventional versus laparoscopic-assisted surgery in patients with colorectal cancer (mrc clasicc trial): Multicentre, randomised controlled trial. Lancet 365(9472): 1718-1726, 2005.

5 Yamamoto S, Inomata M, Katayama H, Mizusawa J, Etoh T, Konishi F, Sugihara K, Watanabe M, Moriya Y and Kitano S: Short-term surgical outcomes from a randomized controlled trial to evaluate laparoscopic and open $\mathrm{d} 3$ dissection for stage ii/iii colon cancer: Japan clinical oncology group study jcog 0404 Ann Surg 260(1): 23-30, 2014.

6 Harris SN, Ballantyne GH, Luther MA and Perrino AC Jr.: Alterations of cardiovascular performance during laparoscopic colectomy: A combined hemodynamic and echocardiographic analysis. Anesth Analg 83(3): 482-487, 1996.

7 Charlson ME, Pompei P, Ales KL and MacKenzie CR: A new method of classifying prognostic comorbidity in longitudinal studies: Development and validation. J Chronic Dis 40(5): 373 383, 1987.

8 Hines RB, Chatla C, Bumpers HL, Waterbor JW, McGwin G Jr., Funkhouser E, Coffey CS, Posey J and Manne U: Predictive capacity of three comorbidity indices in estimating mortality after surgery for colon cancer. J Clin Oncol 27(26): 4339-4345, 2009.

9 Krarup PM, Nordholm-Carstensen A, Jorgensen LN and Harling H: Association of comorbidity with anastomotic leak, 30-day mortality, and length of stay in elective surgery for colonic cancer: A nationwide cohort study. Dis Colon Rectum 58(7): 668-676, 2015.

10 Marventano S, Grosso G, Mistretta A, Bogusz-Czerniewicz M, Ferranti R, Nolfo F, Giorgianni G, Rametta S, Drago F, Basile $\mathrm{F}$ and Biondi A: Evaluation of four comorbidity indices and Charlson comorbidity index adjustment for colorectal cancer patients. Int J Colorectal Dis 29(9): 1159-1169, 2014.

11 Schneider EB, Hyder O, Brooke BS, Efron J, Cameron JL, Edil BH, Schulick RD, Choti MA, Wolfgang CL and Pawlik TM: Patient readmission and mortality after colorectal surgery for colon cancer: Impact of length of stay relative to other clinical factors. J Am Coll Surg 214(4): 390-398, 2012.

12 Charlson M, Szatrowski TP, Peterson J and Gold J: Validation of a combined comorbidity index. J Clin Epidemiol 47(11): 1245-1251, 1994.

$13 \mathrm{Wu}$ CC, Hsu TW, Chang CM, Yu CH and Lee CC: Age-adjusted charlson comorbidity index scores as predictor of survival in colorectal cancer patients who underwent surgical resection and chemoradiation. Medicine (Baltimore) 94(2): e431, 2015.
14 Koppie TM, Serio AM, Vickers AJ, Vora K, Dalbagni G, Donat SM, Herr HW and Bochner BH: Age-adjusted Charlson comorbidity score is associated with treatment decisions and clinical outcomes for patients undergoing radical cystectomy for bladder cancer. Cancer 112(11): 2384-2392, 2008.

15 Robbins JR, Gayar OH, Zaki M, Mahan M, Buekers T and Elshaikh MA: Impact of age-adjusted Charlson comorbidity score on outcomes for patients with early-stage endometrial cancer. Gynecol Oncol 131(3): 593-597, 2013.

16 Saji M, Katz MR, Ailawadi G, Fowler DE, Ragosta M and Lim DS: Predictive value of age-adjusted Charlson co-morbidity index for 1-, 3-, and 5-year mortality in patients requiring transcatheter mitral valve repair. Am J Cardiol 120(2): 309-314, 2017.

17 Tian Y, Jian Z, Xu B and Liu H: Age-adjusted Charlson comorbidity index score as predictor of survival of patients with digestive system cancer who have undergone surgical resection. Oncotarget 8(45): 79453-79461, 2017.

18 Dindo D, Demartines $\mathrm{N}$ and Clavien PA: Classification of surgical complications: A new proposal with evaluation in a cohort of 6336 patients and results of a survey. Ann Surg 240(2): 205-213, 2004

19 Kanda Y: Investigation of the freely available easy-to-use software 'ezr' for medical statistics. Bone Marrow Transplant 48(3): 452-458, 2013.

20 Gerges FJ, Kanazi GE and Jabbour-Khoury SI: Anesthesia for laparoscopy: A review. J Clin Anesth 18(1): 67-78, 2006.

21 Tashiro J, Yamaguchi S, Ishii T, Suwa H, Okada I, Kondo H, Miyazawa M, Shinozuka N and Koyama I: Efficacy of laparoscopic colorectal resection for risk patients with severe comorbidity. Hepatogastroenterology 59(119): 2173-2176, 2012.

22 Ichikawa N, Homma S, Nakanishi K, Kazui K, Kashiwakura S, Ohira M, Tsuji T, Suzuki T, Ishikawa T and Taketomi A: Safety of laparoscopic colorectal resection in patients with severe comorbidities. Surg Laparosc Endosc Percutan Tech 26(6): 503507, 2016.

23 Stocchi L, Nelson H, Young-Fadok TM, Larson DR and Ilstrup DM: Safety and advantages of laparoscopic vs. Open colectomy in the elderly: Matched-control study. Dis Colon Rectum 43(3): 326-332, 2000.

24 Aquina CT, Mohile SG, Tejani MA, Becerra AZ, Xu Z, Hensley BJ, Arsalani-Zadeh R, Boscoe FP, Schymura MJ, Noyes K, Monson JR and Fleming FJ: The impact of age on complications, survival, and cause of death following colon cancer surgery. Br J Cancer 116(3): 389-397, 2017.

25 Abraham NS, Young JM and Solomon MJ: Meta-analysis of short-term outcomes after laparoscopic resection for colorectal cancer. Br J Surg 91(9): 1111-1124, 2004.

26 Reza MM, Blasco JA, Andradas E, Cantero R and Mayol J: Systematic review of laparoscopic versus open surgery for colorectal cancer. Br J Surg 93(8): 921-928, 2006.

27 Evans C, Galustian C, Kumar D, Hagger R, Melville DM, Bodman-Smith M, Jourdan I, Gudgeon AM and Dalgleish AG: Impact of surgery on immunologic function: Comparison between minimally invasive techniques and conventional laparotomy for surgical resection of colorectal tumors. Am J Surg 197(2): 238-245, 2009.

Received November 10, 2017

Revised December 13, 2017

Accepted December 14, 2017 